\title{
Field test of long range terrestrial laser scanner and ground-based synthetic aperture radar for area monitoring in open pit mines
}

\author{
A Fowler RIEGL Laser Measurement Systems GmbH, Austria
}

A Geier RIEGL Laser Measurement Systems GmbH, Austria

\begin{abstract}
Slope Stability Monitoring (SSM) has improved dramatically over the past few years with the introduction of total stations, radar and other advanced technologies. This trend is continuing with the use of Terrestrial Laser Scanners (TLS) to enable three-dimensional measurement of slope movements. With useful range of the instruments typically limited by the balance of eye-safety and laser power, the application of $T L S$ systems has been limited to short range scenarios. However, applying infrared wavelengths and novel innovations, these limitations have been surmounted and now monitoring applications beyond 2,000 $m$ are possible at high measurement rates without the safety hazards associated with typical Class $3 R$ long-range laser scanners (LR-TLS). It is now possible to use LR-TLS technology effectively for long range SSM and surface deformation analysis.

In a cooperative field test conducted by RIEGL LMS, DMT GmbH \& Co. KG, and RWE Power AG, a RIEGL VZ-4000 online-waveform processing LR-TLS instrument was deployed in a large opencast coal mine in Germany. LR-TLS data was continuously acquired for a period of 48 hours concurrently with an IDS IBISFM long range ground-based Interferometric Synthetic Aperture Radar (GB-InSAR) system and, additionally, a Leica total station monitoring system. Results from this field test demonstrate the potential of this new $L R$-TLS sensor technology and are compared with the results from those achieved via the GB-InSAR, LR-TLS and Total Station systems to determine the level of usability in active long-range mining situations.
\end{abstract}

\section{Introduction}

Mountains crumble, hills erode, and cliffs tumble into the sea. This process has been occurring since the beginning of time but never before has it been as important as it is today. With the advancement of civilisation into and onto such active surfaces, the importance and value of monitoring these surfaces for deformation is becoming ever more salient.

Remote sensing technologies are actively employed in detecting and quantifying such movements. The ability to optimise warning time before such events occur and to develop an understanding of the mechanisms involved, is determined in large part, by the accuracy, frequency and density of the spatio-temporal aspects of the measurements acquired during the events. Detection of larger movements is possible from satellite-borne remote sensing technologies, but more refined spatial and temporal resolutions are required for detecting and quantifying surface deformations on a smaller scale (Atzeni et al. 2015).

Terrestrially-based active Remote Sensing technologies provide the ability to acquire the necessary level of spatio-temporal data at a resolution needed for tracking slope deformation in real-time. A number of these technologies are employed in slope deformation monitoring, with Interferometric Synthetic Aperture Radar (GB-InSAR) and Tachymeters combined with prism networks forming the primary sources of such datasets. However, GB-InSAR and Robotic Total Station Prism networks leave a gap in information; highly detailed and accurate surface modelling of deformation in 3 dimensions. GB-InSAR provides the ability to produce high-frequency 2D image sequences of deformation, but for spatial reference, these images require projection onto another reference surface, such as a DEM, or similar model (Atzeni et al. 2015). The Robotic 
Total Stations provide precise 3D positions, but is limited to sparsely populated, finite reference points in a prism network.

Long Range Terrestrial LIDAR Scanning (LR-TLS) technology has the potential to fill this gap in technology: providing high density spatio-temporal datasets collected in small time intervals (Reetz \& Gaisecker 2011). While short-range terrestrial LIDAR scanning technology has been around for approximately 20 years, systems achieving reliable data beyond $2 \mathrm{~km}$ have only been introduced in the past few years (Gaisecker et al. 2012). As the technology is still quite new, testing is needed to ensure it provides satisfactory results for this application.

\section{Installation}

The field test consisting of an IDS IBIS-L GB-InSAR system, a RIEGL VZ-4000 LR-TLS and a Leica Tachymeter with prism network was conducted on a section of the RWE Hambach open pit mine near Cologne, Germany in September 2014. The test field was composed of clay and soft rock with bench angles of roughly $10^{\circ}$. The instruments were installed in an advantageous position to balance the range, perspective and region of interest requirements. The test ran for approximately 2.5 days of nearly uninterrupted observation; from 22-24 September 2014. In the course of the test, continued rainfall resulted in some localised slope movements which were detected by multiple systems. The quantity of change and resulting difference map for these movements were produced and a basic report on the result from each system examined.

The installation of each of the systems was as follows (Figure 1):

- The GB-InSAR system was housed in a shipping container and mounted on concrete blocks. An aperture in the side of the shipping container provided an unobstructed view of the test site.

- The LR-TLS instrument was mounted on a steel column which protruded from a $1 \mathrm{~m}$ cube of concrete with two holes for transport by forklift. The mount was installed between the GB-InSAR and Tachymeter instruments, approximately $3 \mathrm{~m}$ from each.

- The Tachymeter was installed within a glass shield and on top of an approximately $2.5 \mathrm{~m}$ tall concrete monument which was submerged approximately $1 \mathrm{~m}$ under the ground surface.

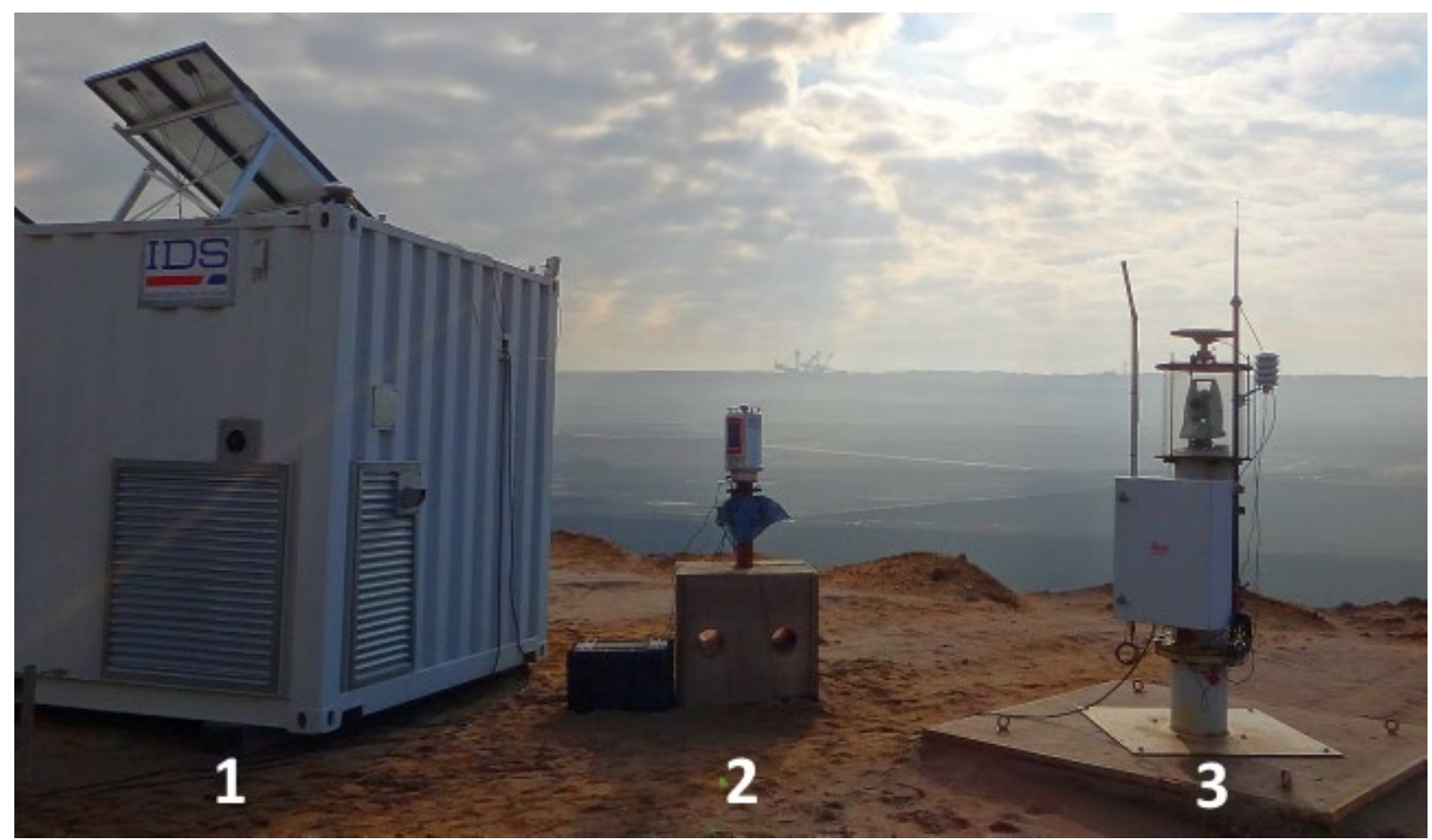

Figure 1 Installation of GB-InSAR (1), LR-TLS (2), and Tachymeter (3) with Test Field in background 


\section{$3 \quad$ Configuration and data processing}

All sensors were configured to optimise range and resolution performance. The manufacturer's respective software packages for each system were employed to produce the best possible results. The results revealed the different levels of sophistication for each of the software solution accompanying the technologies. The GB-InSAR was equipped with a well-developed software package specifically tailored to this application, which provided real-time processing and analysis. For the LR-TLS, a post-processing approach was decided on whereby data would be run through an algorithm to simulate real-time processing. The final results from each system were then compared.

The project was a joint venture and one team operated the GB-InSAR system while the other operated the LR-TLS system. No results were exchanged until all processing had been completed. Thus, each dataset and the results achieved were fully independent.

\subsection{GB-InSAR configuration}

The GB-InSAR system was configured to acquire data on a recurring 5 minute interval with the resulting field-of-view (FoV) parameters as delineated in Table 1. All data was automatically processed in near-real-time by the manufacturer's supplied software and displacement values exported for later comparison.

\section{Table $1 \quad$ GB-InSAR parameters}

\begin{tabular}{cc}
\hline Parameter & Applied value \\
\hline Horizontal field of view & $60^{\circ}$ \\
Vertical field of view & $60^{\circ}$ \\
Range resolution & $\sim 0.75 \mathrm{~m}$ \\
Azimuth resolution & $\sim 4.4 \mathrm{~m} \mathrm{@} 1,000 \mathrm{~m}$ \\
Maximum range & $4,000 \mathrm{~m}$ \\
\hline
\end{tabular}

\subsection{LR-TLS configuration}

The LR-TLS system was configured to automatically acquire data on a fixed 10-minute interval with the parameters in Table 2.

In addition to the scheduled 10-minute scanning interval, the scanner was set up to automatically acquire a network of 10 reflectors every two hours. The process of acquiring all 10 reflectors required approximately 10 minutes each interval, which resulted in the forfeit of one scan sequence every two hours. Aside from initial configuration, all data was acquired by the LR-TLS system autonomously.

\section{Table 2 LR-TLS parameters}

\begin{tabular}{cc}
\hline Parameter & Applied value \\
\hline Horizontal field of view & $90^{\circ}$ \\
Horizontal step-width (resolution) & $0.014^{\circ}$ \\
Vertical field of view & $20^{\circ}$ \\
Vertical step-width (resolution) & $0.018^{\circ}$ \\
Maximum range & $4,000 \mathrm{~m}$ \\
Pulse repetition rate & $50 \mathrm{kHz}$ \\
\hline
\end{tabular}




\subsection{LR-TLS data processing}

The LR-TLS data was post-processed after the field test was fully completed. The data was processed using a combination of software tools developed for the test, software provided by the manufacturer for geometric adjustments to each dataset (adjusting roll, pitch and yaw to each complete scan), and in a final step, software provided by the manufacturer for simulated real-time data processing (all datasets were processed automatically with one set of parameters to simulate real-time processing).

\subsubsection{LR-TLS atmospheric scaling correction}

The first step in the post-processing stage was the application of range corrections induced by atmospheric scaling factors. These values were calculated from atmospheric readings acquired by an onsite weather station. The range variances to 10 planar reflectors prior to correction are shown in Figure 2 as grey lines. The standard deviation of all values was $5.1 \mathrm{~mm}$ over all ranges ( $\min =120 \mathrm{~m}, \max =2.4 \mathrm{~km}$ ). The calculated range scaling correction due to atmospheric change (in $\mathrm{ppm}$ ) is overlaid in green.

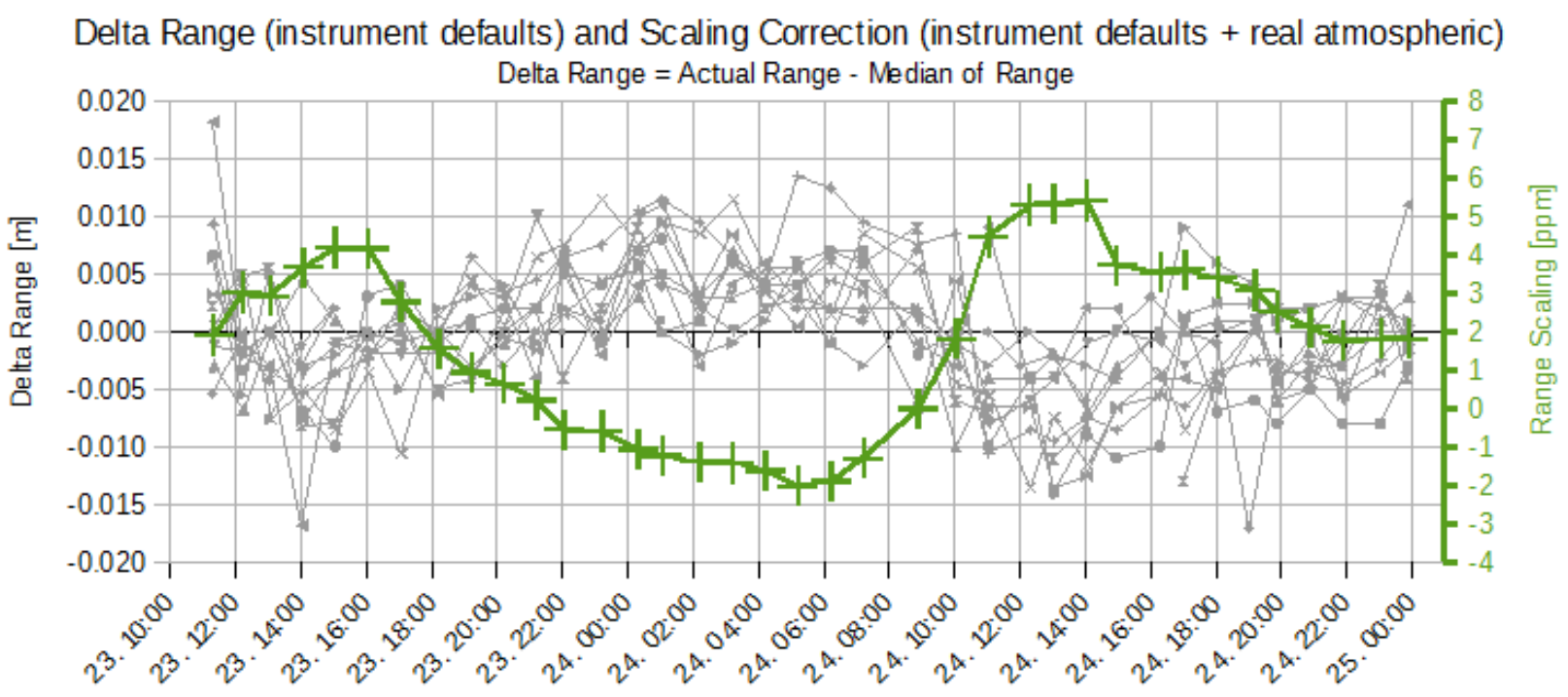

Day of September 2014. Time

Figure 2 Graph showing range variances (in meters) to measured TLS reflectors over time (grey lines) overlaid with calculated atmospheric scaling correction in parts-per-million (green line with crosses)

With the atmospheric range corrections applied, the relative differences in range were again charted, resulting in a final standard deviation of $3.7 \mathrm{~mm}$. The final result can be seen in Figure 3.

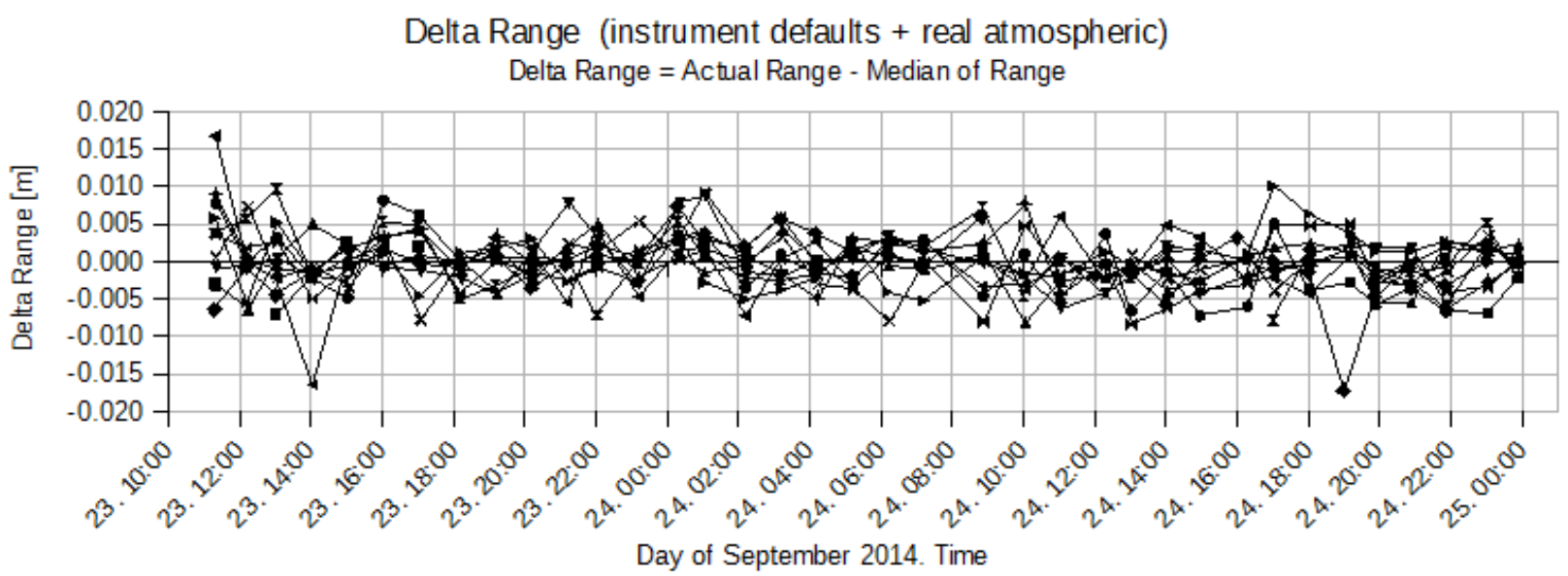

Figure 3 Graph showing resulting range variances to measured TLS reflectors over time AFTER calculated scaling correction was applied (each reflector is represented by a black line) 


\subsubsection{LR-TLS data adjustment}

Persistent operation of LR-TLS systems in active mining environments presents a few challenges. The instrument may be disturbed by machinery in the immediate vicinity (as was the case during this test), blasting or any other number of disturbances. Likewise, moderate rainfall, geological rebound of the mine, and other natural influences can cause minor changes to the material under the concrete mount. Thermal expansion and contraction of the steel beam of the LR-TLS mount according to which side the sun heats also incurs variation to the position and (more significantly) orientation of the instrument. Therefore, a method of registering the datasets together to adjust these errors is required.

Two approaches were tested to determine an optimal method for adjustment. The first method invoked was a least-squared adjustment of the observed values on the reflector network (acquired every two hours). For validation, a second adjustment was performed utilising an iterative closest point (ICP) algorithm on planes extracted from each scan from each epoch with the first scan held as a reference for all others. The ICP algorithm is also known as MSA in the software used for adjustment. The results of each independent method are presented in Figure 4. Note the dramatic influence of the machinery between 23. 07:00 and 23. 17:00.
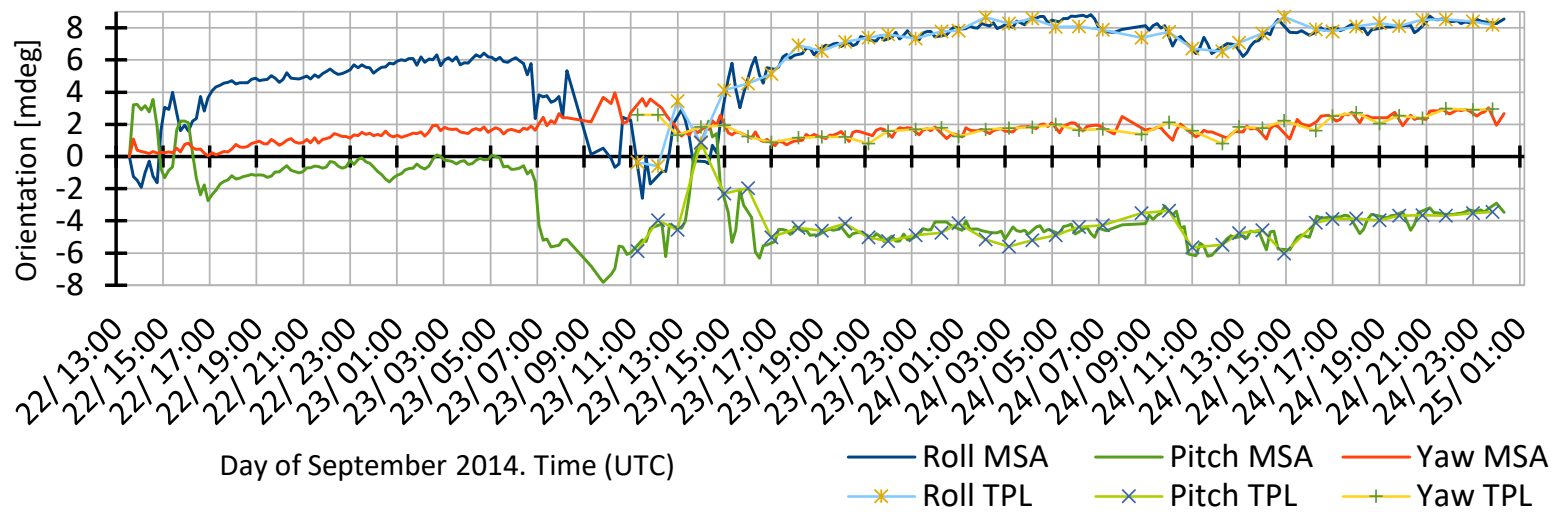

Figure 4 Comparison of LR-TLS Orientation Results from the Reflector Network (TPL) and ICP Adjustment (ICP)

\subsubsection{LR-TLS deformation analysis}

The final analysis used to derive deformation values was completely automated. Parameters were established to optimise results and the automated process was started.

The process operates in the following manner:

- The objective surface was modelled via a 2.5D Raster methodology. Each scan was divided into seven segments, with an optimum reference plane defined for each segment. Each of these planes served as the base of a rasterised grid. Each raster cell of each plane was projected through the point cloud data and all points contained within each raster were averaged to obtain a single height above the plane. The result is a $2.5 \mathrm{D}$ value for each cell (Figure 5).

That raster information for each epoch was then compared to the reference epoch by differencing the raster distance values for each cell along the normal direction of the reference plane. Any cell containing less than was filtered to eliminate poor results causes by insufficient data. 


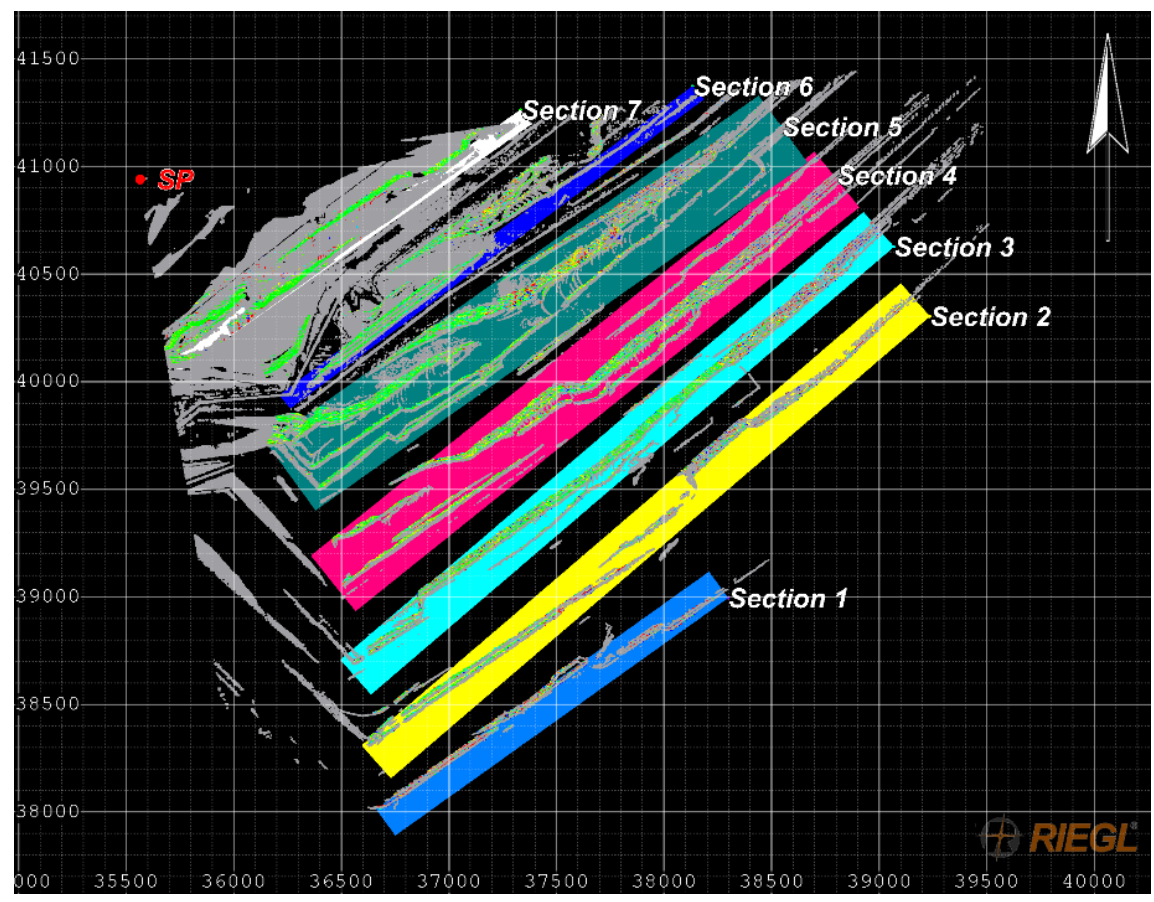

\section{Figure 5 LR-TLS raster planes}

\section{$4 \quad$ Results}

During the course of the monitoring period, moderate rain showers occurred which resulted in some minor slope deformation in a number of locations within the test field (Figure 6). The extent of these deformations was further investigated. However, as there were a number of smaller events, only the most pronounced were investigated. Typical deformations were selected to provide a representative sample of the overall performance for each system.

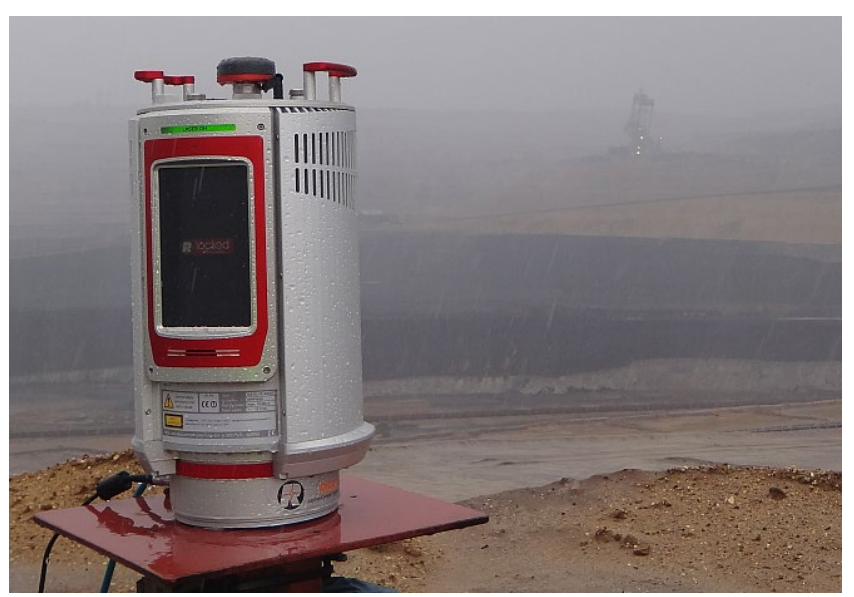

\section{Figure 6 RIEGL VZ-4000 scanning in a moderate rainstorm}

The final step in the project, correlating the two datasets, proved to be more challenging than planned. Although the GB-InSAR system was georeferenced using RTK-GNSS to measure the origin and georeferenced corner cube reflectors used to determine the orientation, assimilating the GB-InSAR and LR-TLS datasets proved to be a challenge due to the large beam size and relative orientation errors encountered with the GB-InSAR system.

In contrast, the LR-TLS system was relatively easy to precisely georeference provided by its integrated sensors: inclination, GNSS, and precise reflector scanning capabilities (see Figure 3 for range measurement 
errors to multiple reference targets). The resulting positioning accuracy of the LR-TLS data was at the centimeter level.

However, with a bit of trial and error, the shift between the GB-InSAR and LR-TLS datasets was found to be around $20-30 \mathrm{~m}$, depending on the range. The challenge this presented was simply one of logistics; the size of the slope deformations often were not larger than $20-30 \mathrm{~m}$ and therefore positively identifying displacements between systems via geodetic coordinates was achieved only after a great deal of coordination between datasets.

\subsection{System coverage}

Both instruments are specified to achieve measurement performance up to 4,000 meters. As the site was limited to roughly 3,000 meters, the ability to test this aspect of performance was not available. The immediate difference between the two technologies is evident in the Laser Scanner's ability to scan a configurable $360^{\circ}$ window, while the GBInSAR system is limited to $60 \times 60^{\circ}$. This creates a striking difference when comparing coverage of each system (see Figure 7) as the LR-TLS was configured to acquire a $90^{\circ}$ horizontal window. The LR-TLS system easily covered the same region as the GB-InSAR system and extended well beyond in the horizontal aspect. While the LR-TLS system covered a much wider Field of View, it required twice as much time (10 minutes) as the GB-InSAR system to do so. There is a direct tradeoff between the configured Field of View and required scan time for the Laser Scanner and this should considered for optimising for different applications in the future.

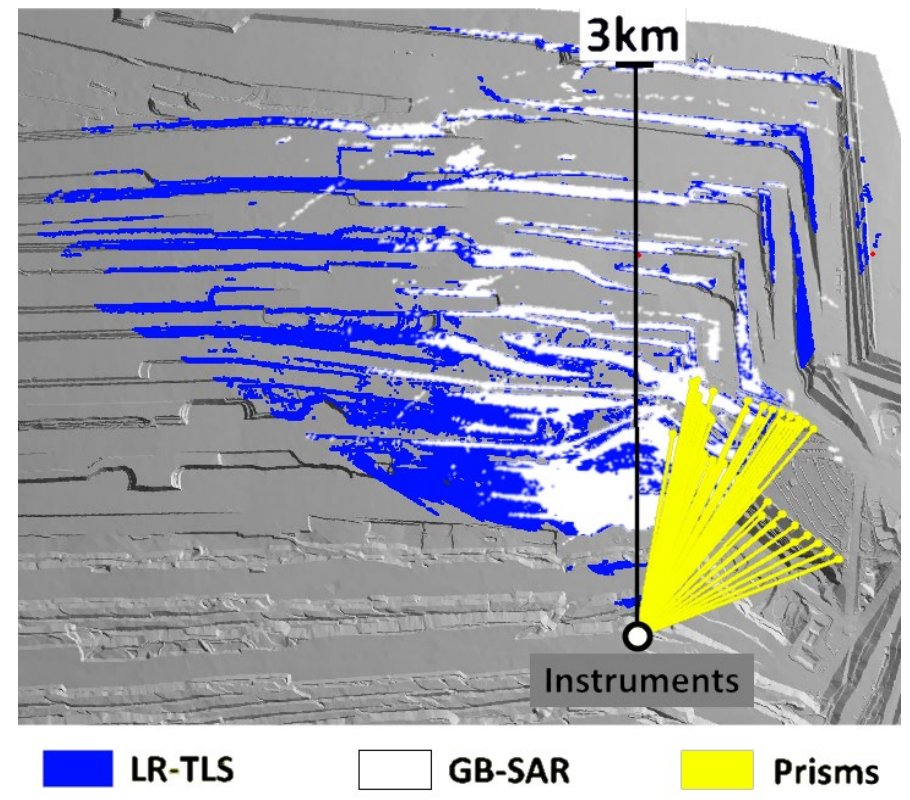

Figure 7 Sensor coverage map. Note: GB-InSAR (white) overlaps LR-TLS (blue)

\subsection{Detection capabilities}

There is a large difference in the detection capabilities afforded by the two systems. Basic physics dictate the characteristics of each. Radar has a large footprint due to its wavelength, but the benefit of the same wavelength is the ability to resolve relative changes to millimetre precision, given that the surface encountered by the radar beam is relatively planar.

The LR-TLS used in this test provided a large number of discrete points with a much smaller beam diameter (see Table 3) compared with the radar system. However, each point has a precision of $10 \mathrm{~mm}$, which means that the variability of each point will create a noisier result, but a more accurate result due to the nature of the system. 
In principle, the differences (see Table 3) can be summarised as this: GB-InSAR provides precise change detection and LR-TLS provides accurate change detection. The difference is not simply a change in terms, it is the difference between tracking fast and slow moving surfaces. Typically, the precision required to repeatably detect change is determined as the capability to measure and order of magnitude more precise than the change itself. In this case, the InSAR system potentially has the ability to detect changes on the order of $0.1 \mathrm{~mm}$, which means it can reliably detect changes of $1 \mathrm{~mm}$. However, due to the limitation of the technology, rapid deformations result in a complete loss of data integrity. A change has occurred, but it cannot be quantified.

LR-TLS does not suffer this issue as it measures in true 3D. However, the results from this test show that the systems can resolve to approximately $4 \mathrm{~mm}$ (Section 3.1.1), which translates to a detectable change of $40 \mathrm{~mm}$ or greater.

Table 3 System characteristics (Sources: IDS 2012 and RIEGL 2015)

\begin{tabular}{|c|c|c|}
\hline & IDS IBIS-FM & RIEGL VZ-4000 \\
\hline Maximum range & $4,000 \mathrm{~m}$ & $4,000 \mathrm{~m}$ \\
\hline \multirow[t]{2}{*}{ Precision } & Spec: $<0.1 \mathrm{~mm}$ (SNR dependent) & Spec: 10 mm \\
\hline & Actual results: $0.3-1 \mathrm{~mm}$ & Actual results: $3-5 \mathrm{~mm}$ \\
\hline \multirow{2}{*}{$\begin{array}{l}\text { Measurement } \\
\text { attributes }\end{array}$} & Amplitude and phase & $X, Y, Z, \theta, \phi, r$ \\
\hline & & Amp., reflectance, deviation \\
\hline \multirow[t]{2}{*}{ Beam footprint } & 4.4 m @ 1,000 m & 0.15 m @ 1,000 m \\
\hline & (4.4 mrad azimuth resolution) & (to perpendicular surface) \\
\hline \multirow{2}{*}{$\begin{array}{c}\text { Displacement } \\
\text { detection method }\end{array}$} & 1D line-of-sight & 1D plane raster \\
\hline & & (3D also realisable) \\
\hline
\end{tabular}

\subsection{Detected events}

Four deformation events were selected to represent the diverse conditions and results which were achieved by both systems (although there were several more noted). The range and location of these are depicted in Figure 8 and a side-by-side comparison of events is provided in Table 4 . All images in Table 4 are scaled from -10 to $+10 \mathrm{~mm}$ of displacement.

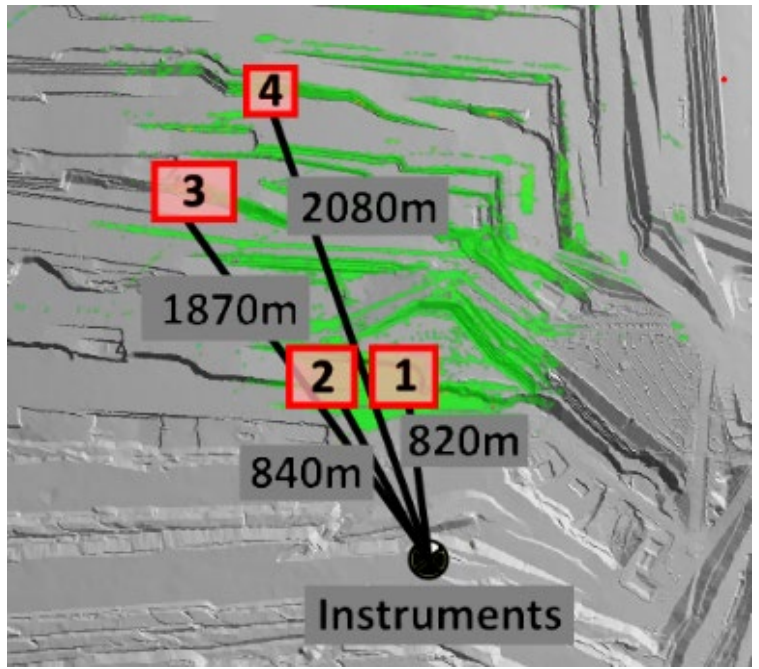

Figure 8 Plot of detected events and their distance from the instruments 


\section{Table 4 Event displacement map comparison}

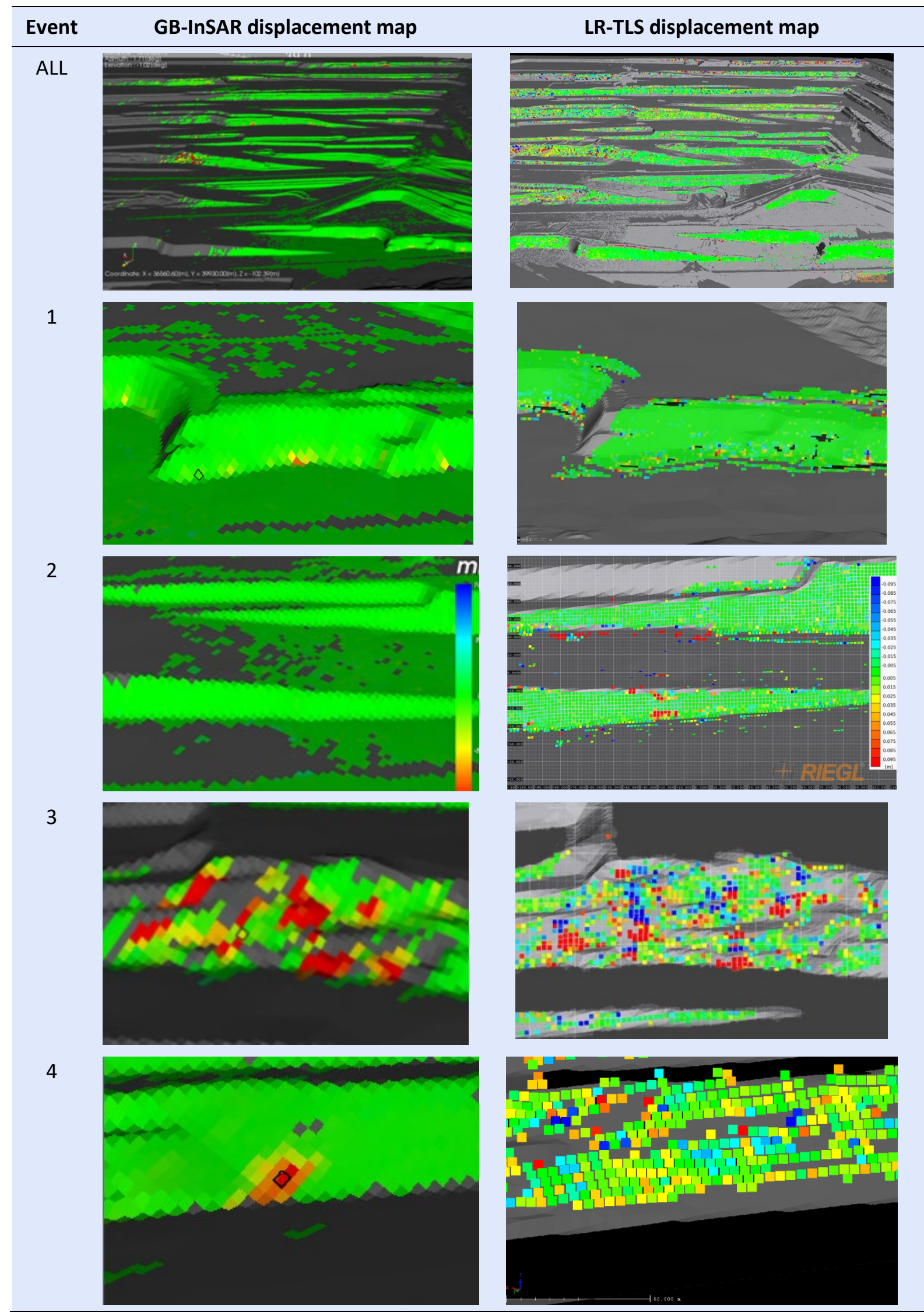




\subsubsection{Event 1}

Both systems detected this event (centre of each image in Table 3, Row 1). The immediately notable difference between each result is the ability of the LR-TLS system to accurately depict erosion and deposition, while the GB-InSAR provides the information that an event has occurred, but does not provide any ability to quantify the erosion and deposition activity (Figure 9).

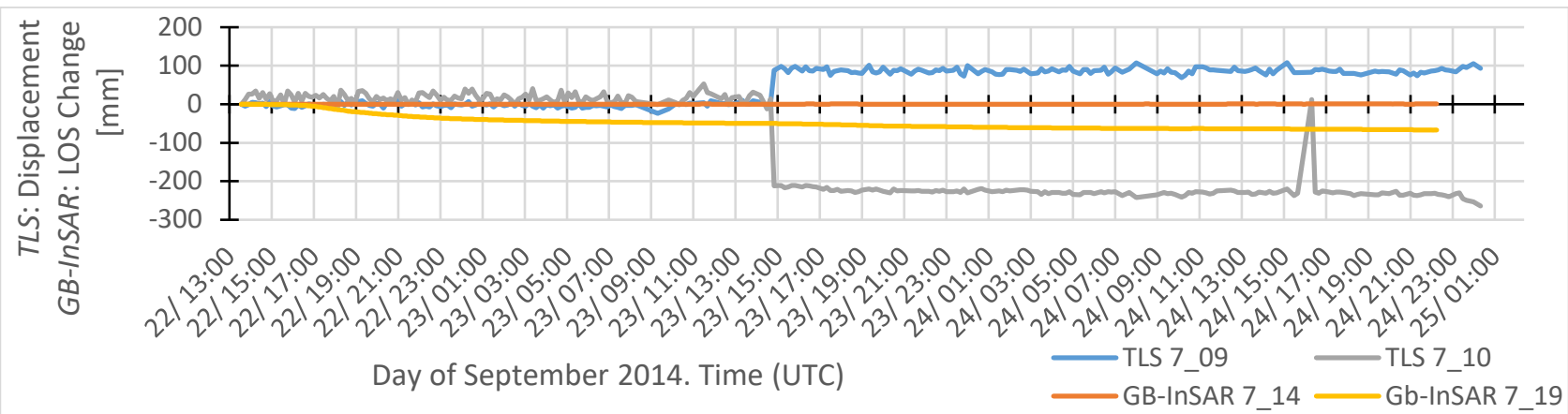

Figure 9 Displacement from 2 cells demonstrating erosion and deposition quantification for GB-InSAR and LR-TLS

\subsubsection{Event 2}

Caused by earth moving equipment, this was technically not a slope failure. It was a machine induced earth movement caused by a dozer pushing earth over an embankment. Displacement graphs from each system are provided in (Figures 10 and 11).

LR-TLS Observed Displacement

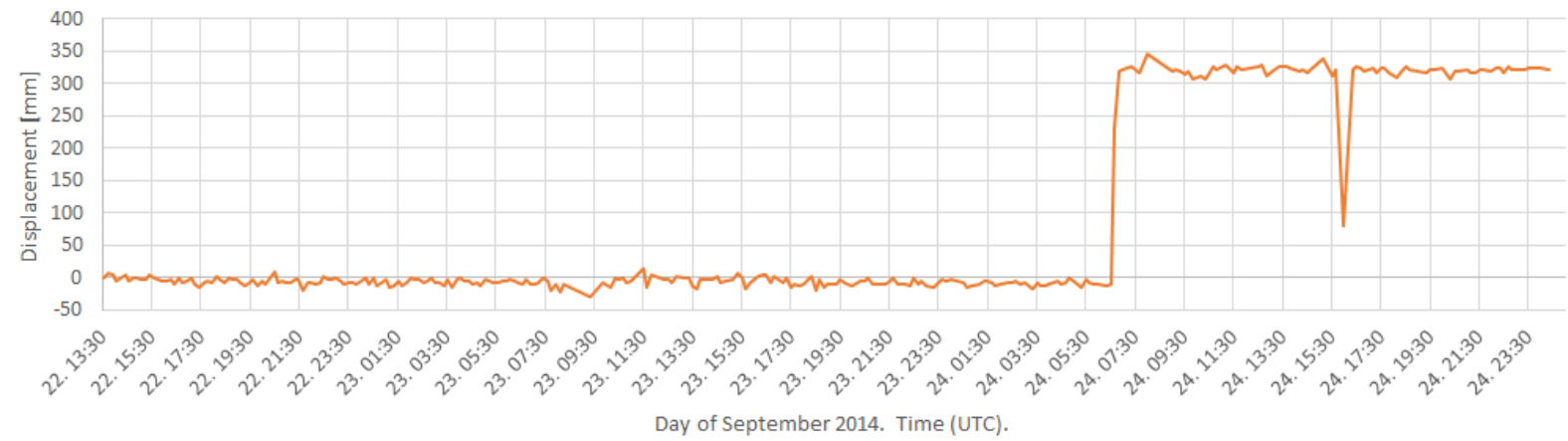

Figure 10 LR-TLS measured displacement of Event 2

InSAR Observed Displacement

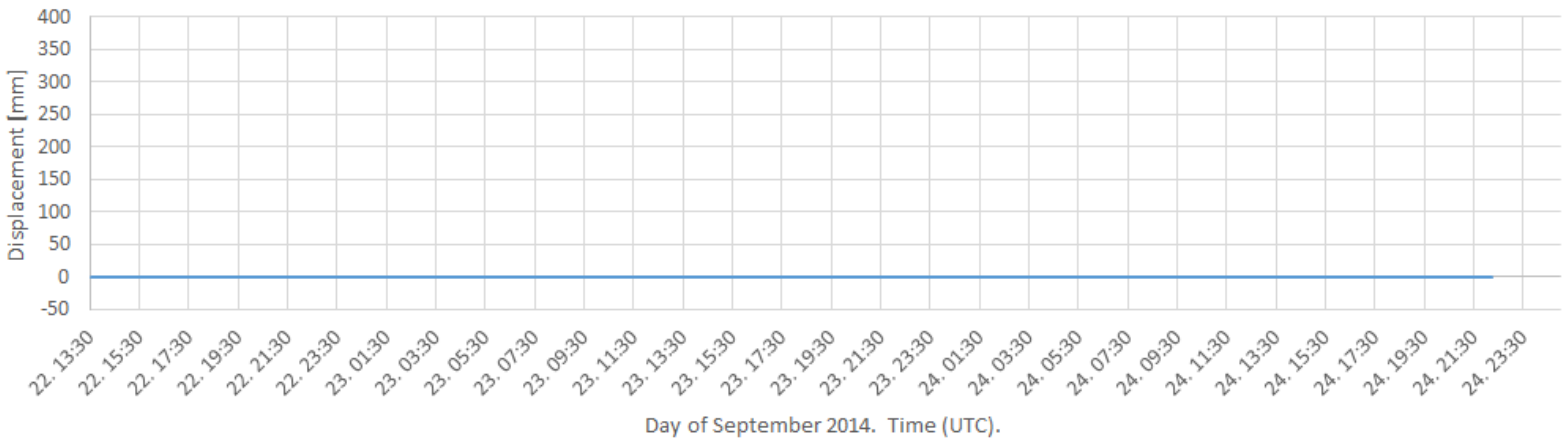

Figure 11 GB-InSAR measured displacement of Event 2 


\subsubsection{Event 3}

This was the largest event recording during testing. A substantial change is readily observable in each of the technology's displacement map from Table 4, Row 3 above. GB-InSAR detected the event(s), but only reported them as deposition events, while the LR-TLS accurately detected both the erosion and deposition event. Extents of the displacement were greater than 3 meters in both deposition and erosion as measured by the LR-TLS system.

\subsubsection{Event 4}

Event 4 appears to be consistent with a traditional earthen creep-type movement. The toe of the highwall is slowly advancing as the crest is slowly subsiding. During the testing period the GB-InSAR detected changes of 50-60 mm, while the LR-TLS results were less conclusive. From the results, it appears that the LR-TLS system was not able to detect the subtle displacement of this system with sufficient precision to render the change as identifiable in the displacement map.

\section{$5 \quad$ Conclusion}

The value of the current level of automation in today's GB-InSAR systems is not to be understated. Once configured, the system operates almost fully automatically (requiring only infrequent inspections). The merits of the technology do not need to be reiterated as they have already been proven in active mine sites around the world (Atzeni et al. 2015). There are, however, limitations to the technology which leave gaps in the ability of geoscientists to accurately model and quantify surface deformations in three dimensions. This aspect of interpretation will prove increasingly vital to the contribution of understanding the mechanics and attributes of soil, rock and material dynamics. This could be overcome if more than one radar would be deployed.

To this extent, the rapid acceleration in LR-TLS developments in recent years has provided a viable means of acquiring highly accurate 4D data in dynamic conditions. The further development and improvement of the automation of these systems will prove critical to their adoption. However, it should be noted that the physical dimensions, performance and rapid improvement of the technology already enables in-depth analysis of slope dynamics with all the benefits of a Remote Sensing technology (safety, ease of deployment, resistance to atmospheric conditions etc.).

It is expected that LR-TLS systems will soon become standard equipment in monitoring applications. Further testing of post-processing and analytical automation will be required to realise a high-performance, high-accuracy real-time LR-TLS monitoring system. Specifically, refined adjustments to counter for physical disturbance of the instrument, better classification and quantification of surface displacement and deformation along with seamless integration with existing infrastructure will be required. The level of automation provided by the RIEGL VZ-4000 enabled the acquisition process to be fully autonomous, which is a must for such isolated installations. With integrated inclination sensors, GNSS and the ability to automatically acquire reflectors, the system provides a straightforward means of georeferencing datasets and therefore simplifies the process of aggregation with other sensor data.

LR-TLS already provides 3D change detection and volumetric change detection capabilities, and is a standard for surveying volumetric changes induced by excavation and other such surface deformation activities (Meinig 2014). It is expected that these capabilities will be extended into the time domain to provide a $4 \mathrm{D}$ record of the mine surface and volumetric changes. As these activities were considered outside the scope of this paper, they have not been detailed. However, these are key considerations for any future research and development.

The fusion of the resulting datasets also provides an additional challenge, as the georeferencing capabilities for Radar data are rather limited due to the physical beam size and lack of internal orientation capabilities. While the radar data was georeferenced to within 5-30 $\mathrm{m}$ (depending on where the data was sampled), attempts to combine the two was made difficult by the ambiguities inherent in the $2 \mathrm{D}$ radar datasets. 
Therefore, it is highly recommended to precisely georeferenced installations in the future to avoid such hassles.

\section{Acknowledgement}

The authors would like to thank RWE for providing a great test field, accommodating all the necessary requirements to fulfil testing, and their cooperation in producing this work.

Additionally, the authors extend their gratitude to DMT for providing their technical expertise in GBInSAR analysis, coordinating the exchange and communication for all participants, companies and providing the fundamental capability of supporting such a technical test involving a topic which is quite sensitive for the region in which it was conducted.

\section{References}

Atzeni, C, Barla, M, Pieraccini, M \& Antolini, F 2015, 'Early warning monitoring of natural and engineered slopes with ground-based synthetic-aperture radar', Rock Mechanics and Rock Engineering, vol. 48, no. 1, pp. 235-246.

Gaisecker, T, Pfennigbauer, M, Sevcik, C, Studnicka, N 2012, 'Terrestrischs Laser Scanning in den Alpen mit dem RIEGL VZ-4000 - für Geländeerfassung, Hangrutschungsüberwachung und Gletschermonitoring', Vermessung \& Geoinformation, pp. 45-52.

IDS Ingegneria dei Sistemi S.p.A 2012, IBIS-L Controller Software, IBIS-L Controller ver. 02.02, User Manual https://www.idscorporation.com/fr/georadar/our-solutions-products/transport/products/item/27-ibis-fl-interferometricradar, Pisa, Italy.

Meinig, H 2014, 'Geotechnische Anforderungen für den Aufbau operativer markscheiderischer Überwachungssysteme im Bereich der Tagebaue der VE Mining AG', Proceedings of Markscheiderisches Kolloquium 2014 Vattenfall Europe Mining AG, Freiberg, Germany.

Reetz, F \& Gaisecker, T 2011, 'Automatisches Deformationsmonitoring an fortschreitenden Tagebauböschungen der Mitteldeutschen Braunkohlen GmbH (MIBRAG) mit RIEGL Laserscan-Technologie' 12. Geokinematischer Tag, Tagungsband. [en: Automatic Deformation Monitoring of Slopes for Mitteldeutschen Braunkohlen GmbH (MIBRAG) with RIEGL Laser Scanning Technology]

RIEGL Laser Measurement Systems GmbH 2015, VZ-4000 Datasheet, RIEGL, Horn, viewed 26 May 2015 , http://www.riegl.com/uploads/tx_pxpriegldownloads/DataSheet_VZ-4000_2015-03-24.pdf 